\title{
Visual marking survives graphical change if meaning is retained
}

\author{
TAKAYUKI OSUGI \\ Chukyo University, Nagoya, Japan \\ AND \\ TAKATSUne KumAda ANd Jun KaWAHARA \\ National Institute of Advanced Industrial Science and Technology, Tsukuba, Japan
}

\begin{abstract}
When some distractors (old items) appear before others (new items) in an inefficient visual search task, the old items are excluded from the search (visual marking). Previous studies have shown that shape changes of static old items are sufficient to eliminate this effect when global luminance is maintained, suggesting that shape identity must be maintained for successful visual marking. It was unclear whether the change in meaning or shape was critical, because these changes were confounded in previous studies. The present study examined whether consistency in the semantic or the graphical identity of old items is critical for visual marking by introducing shape change in the absence of meaning change. The results indicated that visual marking survived graphical changes in old items as long as their meaning was maintained, suggesting that the memory template underlying visual marking represents the semantic identity of old items.
\end{abstract}

One visual function that is frequently used in everyday life is visual search. When we search for a target in a cluttered scene, the visual system uses attention to restrict the search to specific regions or objects, because it is not possible to process the entire scene. Such deployment of attention is achieved in several ways. For example, visual attention may be allocated to objects that appear abruptly (Yantis \& Jonides, 1984) or to objects that differ from background items (Posner, 1980; Theeuwes, 1992, 2004). Attentional allocation is also subject to top-down control based on knowledge (Wolfe, Butcher, Lee, \& Hyle, 2003). Specifically, observers can attend to locations where targets are highly likely to appear, to particular stimulus dimensions or features of targets (Egeth, Virzi, \& Garbart, 1984; Kaptein, Theeuwes, \& van der Heijden, 1995; Sobel \& Cave, 2002), or to objects' appearance (Yantis \& Jonides, 1990).

In the present study, we focused on another type of topdown bias in attentional allocation called visual marking, in which objects that have newly appeared are prioritized over objects that have previously been present in the visual field. This phenomenon has been studied by using visual search tasks. For example, when nontargets in an inefficient search are displayed in two frames, so that the second half of the items (new items) are added to a display containing the first half (old items) and a target always appears in the second frame, search efficiency, as indexed by reaction time $\times$ set size function, is greatly reduced, relative to when all the items appear simultaneously (Watson \& Humphreys, 1997).
Some unique characteristics of the effect of previewing on search efficiency have been delineated in the literature. For example, visual marking can encompass many (more than 30) objects (Jiang, Chun, \& Marks, 2002a). Such a vast capacity contrasts sharply with the fact that a relatively small number of objects ( 3 or 4 ; Yantis \& Jones, 1991) receive priority in the case of attentional capture by abrupt onset. Another important characteristic of visual marking is that it involves active suppression. Specifically, when a probe to be detected appears at the location of an old item, the accuracy for the probe detection is lower than when the probe appears at the location of a new item (Olivers \& Humphreys, 2002; Watson \& Humphreys, 2000). Moreover, a recent study (Kunar, Humphreys, \& Smith, 2003a) revealed that temporal segregation between the onsets of old and new items is not sufficient to produce visual marking. These findings are consistent with the view that active suppression of old items is involved (for alternative suggestions, see Agter \& Donk, 2005; Donk, 2005; Donk, Agter, \& Pratt, 2009; Donk \& Theeuwes, 2001, 2003; Donk \& Verburg, 2004; Jiang, Chun, \& Marks, 2002b; Pratt, Theeuwes, \& Donk, 2007).

One dominant explanation for visual marking is that it is achieved by active suppression of locations of old items via a memory template (Osugi, Kumada, \& Kawahara, 2009; Watson \& Humphreys, 1997). In this scheme, the memory template is updated at the locations of old items in response to bottom-up activity through dynamic visual pathways in the case of changing items. Therefore, visual marking is

T. Osugi, mtaka-oosugi@aist.go.jp 
vulnerable to changes in the old items during the preview period. For example, visual marking disappears when old items suddenly alter their shapes during the previewing period. It is assumed that such abrupt changes evoke bottomup activities at the old locations and thereby send signals to the memory template, resulting in removal of the trace of the old items. Thus, the shape identities of old items in the memory template have to be maintained for visual marking to persist. Watson and Humphreys (2002) showed that visual marking under circumstances in which old and new elements are differently colored is unaffected by luminance or color changes in the old static items but is eliminated by their shape change (for related findings, see also Kunar, Humphreys, \& Smith, 2003b; Watson, Braithwaite, $\&$ Humphreys, 2008). Furthermore, shape changes are also sufficient to eliminate visual marking when global luminance is maintained (Watson \& Humphreys, 2002). Even when a drastic luminance change is introduced by sliding a temporary occluding object over the search display, visual marking is maintained if the shapes of the old items are consistent across the pre- and postocclusion views (Kunar, Humphreys, Smith, \& Watson, 2003). These results suggest that the memory template underlying visual marking includes the shapes of old items.

An important question is whether the critical change affecting visual marking is a change in the shape or in the meaning of the old item, because changes in shape have been accompanied by changes in meaning in previous research. For example, in Watson and Humphreys's (2002) study, the old items, two segment right-angle brackets, were replaced with uppercase $H \mathrm{~s}$, creating both semantic and graphical changes. ${ }^{1}$ The extant evidence merely suggests that visual marking does not occur when the graphical identities of old items change during the preview. This, however, does not necessarily mean that the memory template involved in visual marking carries only the graphical identities of old items. Rather, at least two possibilities arise regarding the nature of the memory template. One possibility is that the template includes only the graphical identities of old items and monitors consistency of these identities during the preview period. Once the graphical consistency is breached, the visual system resets the contents of the memory template and ceases to maintain suppression of the old items. Alternatively, it is possible that the template includes both the graphical and semantic identities of the old items. In this scenario, visual marking would survive even after graphical changes, as long as the old items were semantically consistent across the pre- and postchange views.

In the present study, the contents of the memory template responsible for visual marking were examined by questioning whether the maintenance of the semantic identities of old items is sufficient for the preview advantage in search efficiency to occur. We measured the reaction times required to search for a target and used search slope as a function of set size as an index of the prioritized selection of new over old items. In the present study, we introduced three types of shape change: lower- and uppercase letter change in Experiment 1A, numeral-word change in Experiment 1B, and picture-word change in Experiments 2A and 2B.

\section{EXPERIMENT 1A}

In Experiment 1A, we compared the search functions in two conditions. In the consistent condition, the old items, lowercase letter $t \mathrm{~s}$, were replaced with uppercase $T \mathrm{~s}$, creating a graphical change while maintaining meaning. In the inconsistent condition, the old items, lowercase letter $f \mathrm{~s}$, were replaced by uppercase letter $T \mathrm{~s}$, creating both a semantic and a graphical change. Upon these changes, new items (uppercase letter $L \mathrm{~s}$ ) appeared at blank locations. As a control condition, we used a display in which all the items appeared simultaneously. Slope would be expected to be shallower when visual marking occurs than in the control condition. If the memory template includes only the graphical identity, the search slopes in the consistent and inconsistent conditions will be comparable to each other and equivalent to that in the control condition. In contrast, if the memory template includes semantic representation of the old items and if preservation of this representation is sufficient, the slope in the consistent condition will be shallower than that in the other two conditions.

\section{Method}

Participants. Nineteen undergraduate students (14 males, 5 females; mean age, 21.0 years) from the participant pool of the National Institute of Advanced Industrial Science and Technology (AIST, Tsukuba, Japan) participated for pay. All reported normal or corrected-to-normal visual acuity and normal color vision. They were all also very familiar with English alphabet characters.

Stimuli. The stimuli were displayed on a CRT monitor (Multiscan G220, Sony) controlled by a computer operating Microsoft Windows, MATLAB, and Psychophysics Toolbox (Brainard, 1997; Pelli, 1997). The viewing distance was approximately $60 \mathrm{~cm}$. The stimuli consisted of green lowercase letter $t \mathrm{~s}$ and $f \mathrm{~s}$ subtending $0.58^{\circ}$ in height and $0.38^{\circ}$ in width and green uppercase letter $T \mathrm{~s}$ and $L \mathrm{~s}$ subtending $0.58^{\circ}$ in height and $0.58^{\circ}$ in width. The luminance of these letters was $3.1 \mathrm{~cd} / \mathrm{m}^{2}$. The target was an uppercase $L$ that was rotated clockwise $90^{\circ}$ or $180^{\circ}$, whereas the distractors were upright uppercase letter $L$ s and those rotated $270^{\circ}$, as well as upright capital letter $T \mathrm{~s}$. The preview items were upright lowercase letter $t \mathrm{~s}$ and $f \mathrm{~s}$. The items were presented at randomly selected intersections of an imaginary spiderweb grid. The spiderweb was constructed of 8,16 , or 24 equidistant arms and three concentric circles with radii of $1.3^{\circ}, 2.74^{\circ}$, and $4.18^{\circ}$ (see Figure 1 ). A red $\left(2.3 \mathrm{~cd} / \mathrm{m}^{2}\right)$ fixation cross $\left(0.9^{\circ} \times 0.9^{\circ}\right)$ was presented in the center of the display.

Design and Procedure. Two independent variables were manipulated within observers: meaning (three levels: consistent, inconsistent, and control) and set size (two levels: 4 and 16). At the beginning of the experiment, the participants were informed about the nature of the search task. The participants completed six blocks of trials (two blocks each for the three search types counterbalanced across participants). Each block consisted of a total of 40 trials ( 20 for each set size, mixed within a block). Experimental blocks were preceded by a practice block consisting of 40 trials.

A trial began with the presentation of a fixation cross for $500 \mathrm{msec}$. In the consistent condition, the old items (lowercase $t \mathrm{~s}$ ) appeared for $1,000 \mathrm{msec}$ and then were replaced with uppercase $T \mathrm{~s}$. In the inconsistent condition, the old items (lowercase $f \mathrm{~s}$ ) were replaced by uppercase $T \mathrm{~s}$. Upon these changes, new items (uppercase $L \mathrm{~s}$ ) appeared at blank locations. New distractors consisted of homogeneous letters (either upright uppercase letter $L s$ or those rotated $270^{\circ}$ ). In the control condition, uppercase $T \mathrm{~s}$ and $L \mathrm{~s}$ appeared simultaneously. The participants searched for an uppercase $L$ that had been rotated clockwise $90^{\circ}$ or $180^{\circ}$ and indicated the angle by pressing the " $\mathrm{z}$ " key on the keyboard to indicate $\rceil$ or the " $m$ " key to indicate $\Gamma$ with the left 


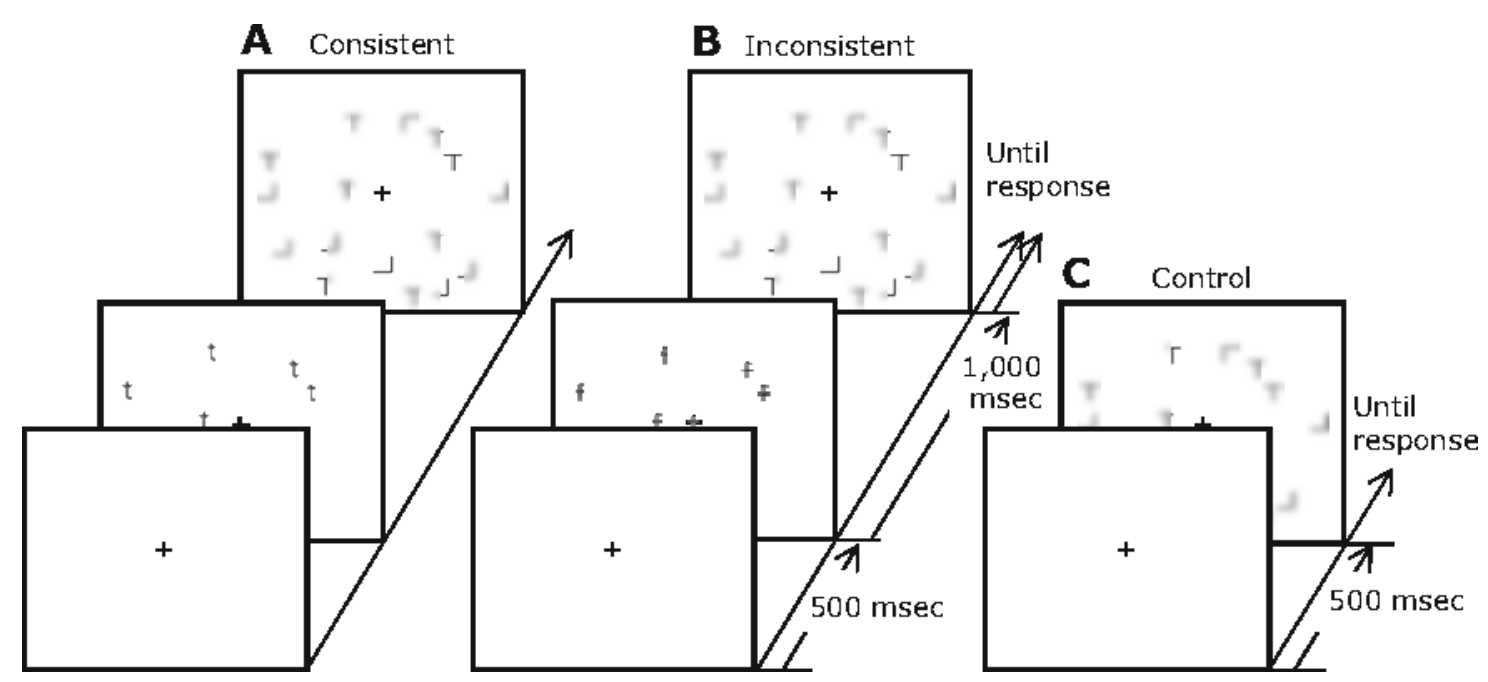

Figure 1. Schematic diagrams of the stimulus sequence in Experiment 1A. (A) The consistent condition. The old items (lowercase $t \mathrm{~s}$ ) were replaced by uppercase $T \mathrm{~s}$, and new items (uppercase $L \mathrm{~s}$ ) appeared. (B) The inconsistent condition. The old items (lowercase $f \mathbf{s}$ ) were replaced with uppercase $T \mathrm{~s}$, and then new items (uppercase $L \mathbf{s}$ ) appeared. (C) The control condition. All items (uppercase $T$ s and $L s$ ) appeared simultaneously.

and right index finger, respectively. Reaction times were measured. When the responses were incorrect or the reaction time was longer than 5,000 msec, a 1000-Hz tone was presented for $20 \mathrm{msec}$.

\section{Results}

Reaction times on error trials and those longer or shorter than \pm 2.5 standard deviations from the mean were excluded from analyses in both this and the following experiments. Figure 2 shows the mean reaction times, and Table 1 shows the mean error rates. An ANOVA on the reaction time data, with meaning (consistent, inconsistent, and control) and set size (4 and 16) as within-subjects factors, identified significant main effects of meaning $[F(2,36)=16.61, p<.01]$ and set size $[F(1,18)=120.01$, $p<.01]$. The interaction between these factors was also significant $[F(2,36)=14.75, p<.01]$. A similar analysis on the error rates indicated that the main effect of set size was significant $[F(1,18)=6.42, p<.05]$. No other main effects or interactions were found.

We conducted three separate two-way within-subjects ANOVAs in which the reaction time data were contrasted between the preview and control conditions. This analysis is commonly adopted in visual-marking studies to compare the effects of search types more closely.

Consistent versus control. The main effects of meaning and of set size were significant $[F(1,18)=30.1, p<$ .01 , and $F(1,18)=118.66, p<.01$, respectively]. The interaction between these factors was also significant $[F(1,18)=32.23, p<.01]$.

Inconsistent versus control. The main effects of meaning and of set size were significant $[F(1,18)=11.75$, $p<.01$, and $F(1,18)=107.07, p<.01$, respectively]. The interaction between these factors was also significant $[F(1,18)=9.16, p<.01]$.

Consistent versus inconsistent. The main effects of meaning and of set size were significant $[F(1,18)=4.47$, $p<.05$, and $F(1,18)=104.67, p<.01$, respectively]. The interaction between these factors was also significant $[F(1,18)=4.62, p<.05]$.

\section{Discussion}

The search slopes in the consistent and inconsistent conditions were shallower than that in the control condi-

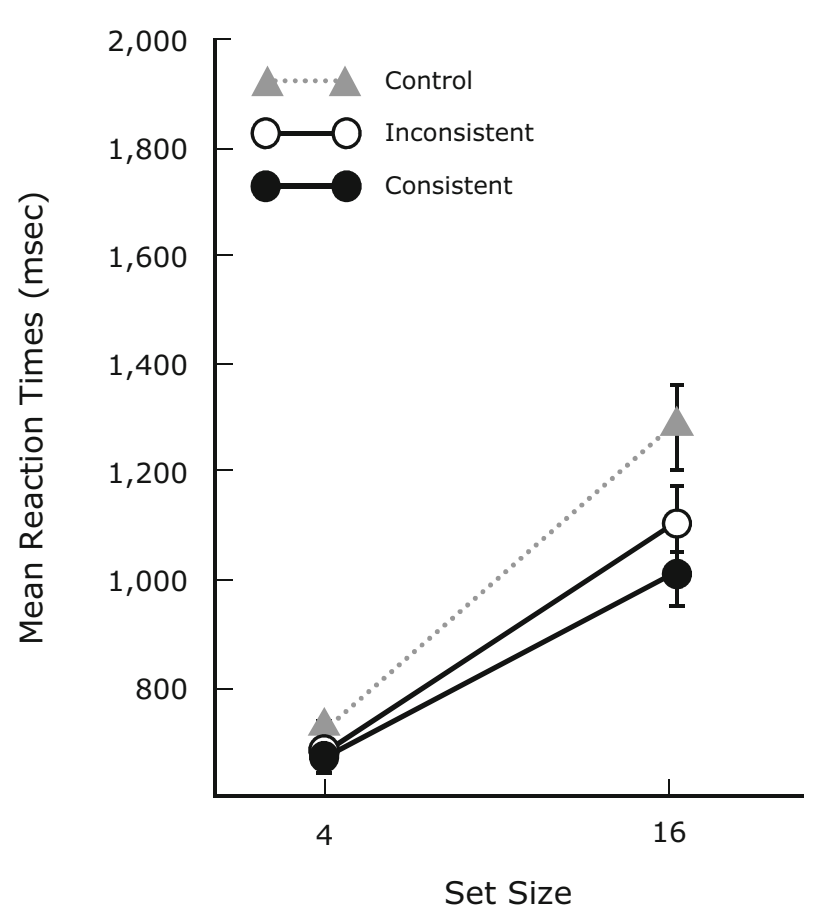

Figure 2. Mean reaction times for visual search as a function of set size in the consistent, inconsistent, and control conditions in Experiment 1A. 
Table 1

Mean Error Rates (\%) in Experiments 1-4

\begin{tabular}{clccc}
\hline & & \multicolumn{3}{c}{ Set Size } \\
\cline { 3 - 4 } Experiment & Condition & 4 & 8 & 16 \\
\hline \multirow{2}{*}{$1 \mathrm{~A}$} & Consistent & 5.6 & & 3.2 \\
& Inconsistent & 4.1 & & 2.8 \\
& Control & 4.3 & & 4.4 \\
$1 \mathrm{~B}$ & Consistent & 3.1 & & 3.8 \\
& Inconsistent & 3.7 & & 5.2 \\
& Control & 3.8 & & 6.0 \\
$2 \mathrm{~A}$ & Consistent & 2.7 & & 5.2 \\
& Inconsistent & 2.9 & & 3.8 \\
& Control & 3.2 & & 5.4 \\
$2 \mathrm{~B}$ & Consistent & 1.6 & & 2.8 \\
& Inconsistent & 1.5 & & 2.9 \\
& Control & 2.2 & & 3.6 \\
3 & Consistent & 3.6 & & 5.3 \\
& Inconsistent & 2.8 & & 6.6 \\
& No change & 4.4 & & 5.8 \\
& Control & 2.1 & & 7.7 \\
& Consistent & 5.0 & 2.8 & 4.7 \\
& Inconsistent & 5.0 & 6.7 & 8.1 \\
& Control & 4.5 & 5.2 & 8.2 \\
\hline
\end{tabular}

tion. This indicates that previewing some items was advantageous in searching for the forthcoming items in the consistent and inconsistent conditions. Such an advantage is consistent with a typical visual-marking effect (Watson \& Humphreys, 1997). More important, the analysis of the search slopes indicated that the search was more efficient in the consistent condition than in the inconsistent condition. This result indicates that visual marking was more vulnerable to the change that included both graphical and semantic identities (i.e., the inconsistent condition), as compared with only the graphical identity (i.e., the consistent condition). The finding that consistency in the semantic identity of the old items aids efficient search implies that the memory template employs meaning as one factor in visual marking.

The results reveal that the search slope in the inconsistent condition was steeper than that in the consistent conditions but still was shallower than that in the control condition. This result indicates a partial contribution of graphical consistency to visual marking. In other words, if the effect of visual marking was dominated by graphical and semantic consistencies, the effect should have disappeared completely in the inconsistent condition. The residual marking effect observed in the inconsistent condition suggests that the mere presence of objects in the same locations throughout the trial would contribute to the visual-marking effect (Watson \& Humphreys, 1997). This finding seems inconsistent with a finding reported by Kunar et al. (2003b); they showed that a within-category change from one letter to another (from a $Z$ to an $H$ ) fully disrupted marking of static items. One could argue that this apparent inconsistency derives from the differences in magnitude of the changes across the displays: The greater the change that occurs between the displays, the more the marking effect suffers from its disruptive impact. Specifically, the pre- and postchange letters used by Kunar et al. (2003b) were completely dissimilar (the $Z$, which consisted of two horizontal strokes and one diagonal stroke, was replaced with the $H$, which consisted of two vertical strokes and one horizontal stroke). Transitions between such dissimilar features introduced a large change, resulting in total disruption of the marking effect. In contrast, the magnitude of change introduced in the present experiment was relatively moderate (e.g., the $f$, which consisted of a pair of vertical and horizontal strokes, was replaced with the $T$, which consisted of the same features). Therefore, the visual-marking effect was not eliminated completely. If this line of reasoning were in fact the case, introducing a rather drastic change across the displays would abolish the marking effect. We tested this possibility in Experiment 1B.

\section{EXPERIMENT 1B}

In this experiment, we introduced a new set of stimuli. We compared the search efficiencies when participants searched for the tilted Japanese kanji character 四 (meaning four in English) among upright 四s and tilted 五s (five in English). In the consistent condition, each Arabic numeral 5 turned into 五 (five). Upon these changes, new distractors, 四s (four), and a target, 四, appeared at blank locations. In the inconsistent condition, Arabic numeral $2 \mathrm{~s}$ were replaced by 五s.

\section{Method}

Another sample of 13 undergraduate students (10 males, 3 females; mean age, 20.8 years) from the AIST participant pool participated for pay. The stimuli, apparatus, and procedures were the same as those in Experiment 1, with the following exceptions. The stimuli consisted of gray Arabic numerals representing 2 and 5 and the Japanese kanji characters 四 (four) and 五 (five), each subtending $0.9^{\circ}$ in height and $1.8^{\circ}$ in width. The target was a tilted $\left(15^{\circ}\right.$ or $345^{\circ}$ from the vertical) kanji character $\mathbb{L}$ ( four), whereas the distractors were upright 四s and tilted 五s. The preview items were tilted Arabic $2 \mathrm{~s}$ and $5 \mathrm{~s}$. These items were presented at randomly selected locations on an invisible $5 \times 5$ matrix subtending $8.05^{\circ}$ in height and $11.5^{\circ}$ in width (see Figure 3 ). We changed from the spiderweb grid used in Experiment 1 A to a $5 \times 5$ matrix for Experiment $1 \mathrm{~B}$, to avoid overlap of new stimuli with those in the previous arrangement. In the consistent condition, the old items, $5 \mathrm{~s}$, were presented for $1,000 \mathrm{msec}$ and were then replaced with tilted 五s. In the inconsistent condition, the old items, 2s, were replaced by 五s. Upon these changes, new distractors, 四s, and a target, 四, appeared at blank locations. In the control condition, 五s, 四s, and a 四 appeared simultaneously. In these trials, the participants searched for a tilted 四 and indicated the tilt by pressing the " $\mathrm{z}$ " or " $\mathrm{m}$ " key for a tilt to the left or to the right, respectively.

\section{Results}

The same ANOVA as that conducted in Experiment 1 on the reaction time data (Figure 4), with meaning (consistent, inconsistent, and control) and set size (4 and 16) as withinsubjects factors, identified significant main effects of meaning $[F(2,24)=10.36, p<.01]$ and set size $[F(1,12)=$ $117.36, p<.01]$. The interaction between these factors was also significant $[F(2,24)=12.71, p<.01]$. A similar analysis on the error rates revealed no main effects or interactions. We conducted three separate two-way within-subjects 


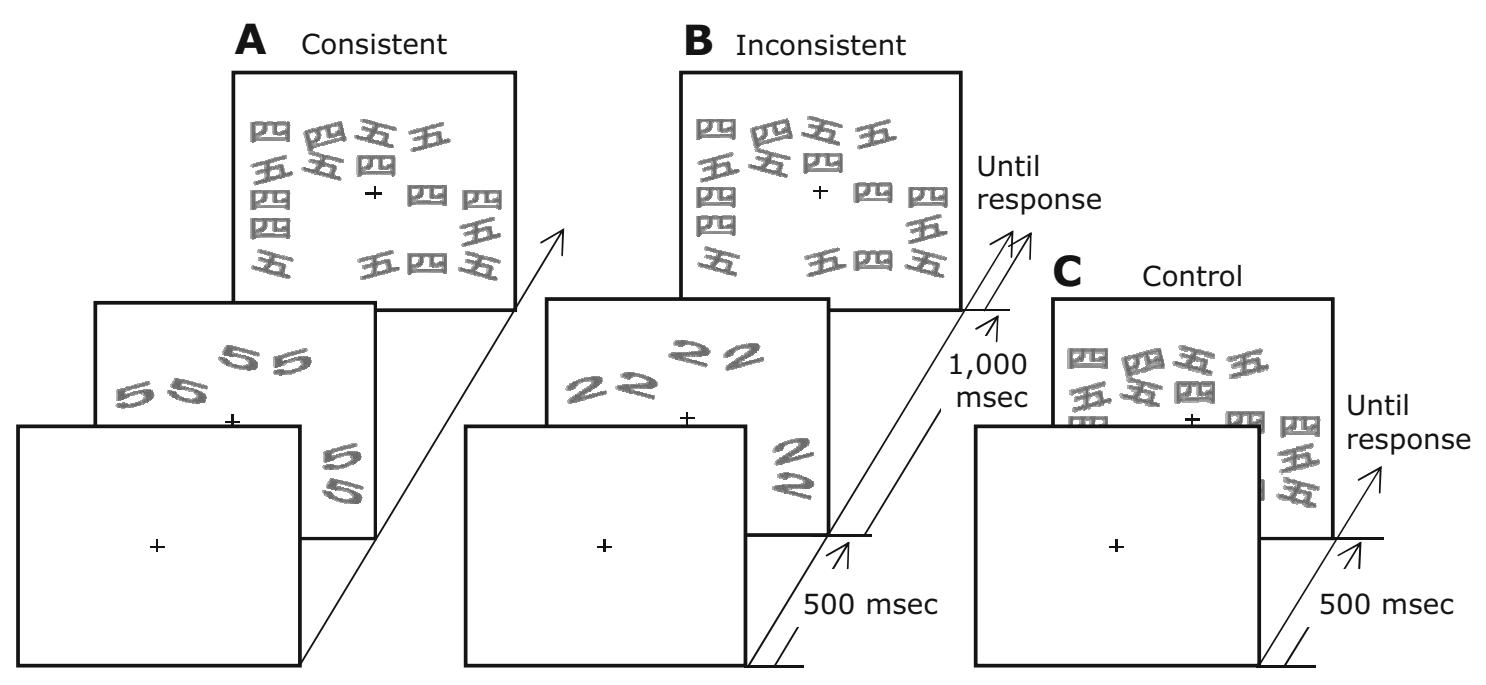

Figure 3. Schematic diagrams of stimulus sequences in Experiment 1B. (A) The consistent condition. The old items (Arabic numeral 5) were replaced with Japanese kanji characters, 五 (five in English), and new items (kanji characters 四, meaning four in English) appeared. (B) The inconsistent condition. The old items (Arabic numeral 2) were replaced with Japanese kanji characters, 五, and new items appeared (四 and a target, 四). (C) The control condition. All items (kanji characters 五, 四, and 四) appeared simultaneously.

ANOVAs, in which the reaction time data were contrasted between the preview and control conditions.

Consistent versus control. The main effects of meaning and of set size were significant $[F(1,12)=15.08, p<$ .01 , and $F(1,12)=108.73, p<.01$, respectively]. The interaction between these factors was also significant $[F(1,12)=21.85, p<.01]$.

Inconsistent versus control. The main effects of meaning and of set size were significant $[F(1,12)=8.67$, $p<.05$, and $F(1,12)=137.77, p<.01$, respectively]. The interaction between these factors was also significant $[F(1,12)=8.23, p<.05]$.

Consistent versus inconsistent. The main effect of meaning did not reach significance $[F(1,12)=1.33, p=$ .27]. The main effect of set size was significant $[F(1,12)=$ $68.86, p<.01]$. The interaction between these factors was also significant $[F(1,12)=4.83, p<.05]$.

\section{Discussion}

An analysis of the search slopes indicated that search was more efficient in the consistent condition than in either the inconsistent or the control condition. The pattern of the results was similar to that found in Experiment 1A, replicating the survival of semantic consistency across the graphical change.

In the present result, the search slope in the inconsistent condition was shallower than that in the control condition. This finding differs from that of Kunar et al. (2003b). One possible explanation for this difference is that the null results in Kunar et al.'s (2003b) study were obtained because the search slope in the control condition was underestimated, since the items in the control condition could be segregated into two groups by color and, thus, subset search by color-based attentional guidance (Egeth et al., 1984; Wolfe, 1994) would reduce the search slope (e.g., Watson \& Inglis, 2007). From this perspective, a search for the target could be restricted to the subset of items even in the control condition. Therefore, the underestimated slope in the control condition might potentially obscure the visual-marking effect, if any, in the inconsistent condition. In fact, the slope in the control (conjunction) condition in Kunar et al.'s (2003b) study (32.3 msec/

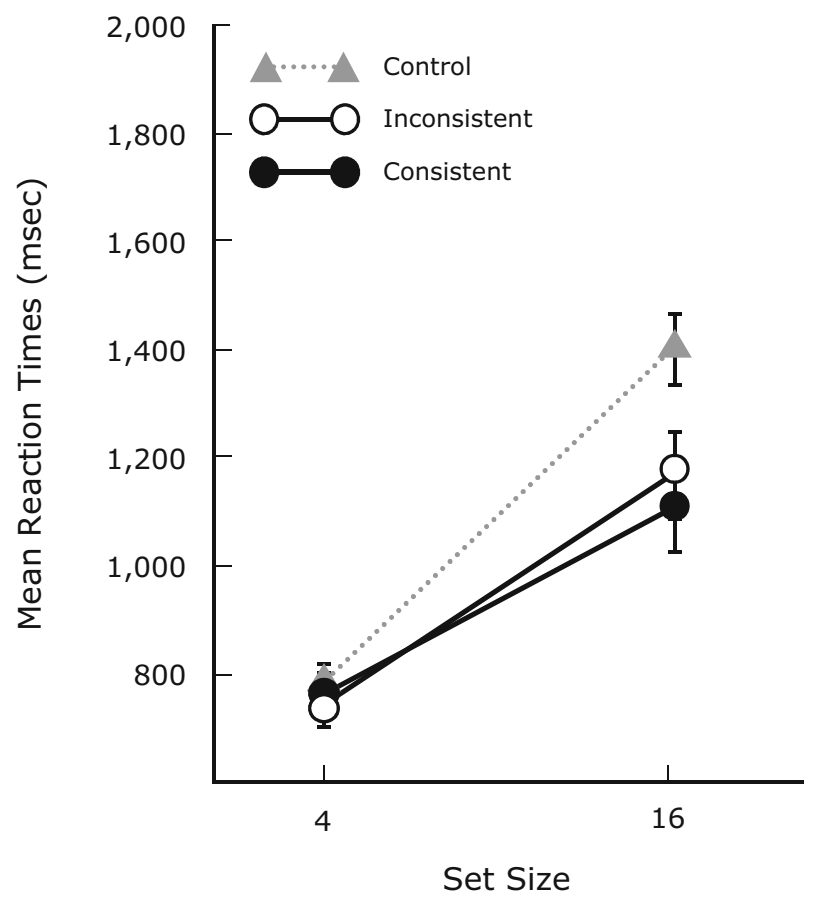

Figure 4. Mean reaction times for visual search as a function of set size in the consistent, inconsistent, and control conditions in Experiment 1B. 
item) was shallower than those in the control conditions in Experiments 1A and 1B of the present study (47.4 and $51.6 \mathrm{msec} / \mathrm{item}$, respectively). This result is consistent with the interpretation above.

Second, it is possible that Kunar et al. (2003b) failed to find the marking effect across a within-category change because of a shape-based grouping between the target and old items. The participants in their study might have perceived the target and old items as being in the same perceptual group because the old items were replaced with the same shapes as the target upon the onset of the new items. In this scenario, the shape-based perceptual grouping between the target and old items disrupts the temporal segmentation that could have occurred when the new items were presented (Jiang et al., 2002b). As a result, the participants were unable to maintain the marking across the change in the shape dimension.

Finally, expectation of target shape might also play a role in the marking of old items. Specifically, Watson and Humphreys (2005) found that the presentation of taskirrelevant disks just before the appearance of new items disrupted the effect of visual marking only when the irrelevant stimuli shared colors with the new items. This result suggests that visual marking is disrupted by the appearance of a new object matching the observer's anticipatory set for new items (see also Braithwaite \& Humphreys, 2003). Given these studies, the null result of Kunar et al. (2003b) might reflect the extra disruption by the adoption of a shape-based anticipatory set for the target because the old items turned into the same shapes as the target.

\section{EXPERIMENT 2A}

Experiments 1A and 1B revealed that the semantic consistency of old items supports visual marking in the absence of graphical consistency. As a caveat, note that the changes applied in the present circumstances were relatively small, in that the items before and after the change both belonged to the alphabet or numeric categories. Another concern is that the change from the letter $f$ to the letter $T$ or that from the digit 5 to the kanji character 五 (five) could be merely phonological, rather than semantic.

To test the generality of semantically supported visual marking, we introduced a new set of stimuli in Experiment 2 . We compared search efficiency while participants searched for a dog among cows. During the preview in the consistent condition, the cows were represented by pictorial images. Upon the onset of new items at blank locations, each old item turned into a Japanese kanji character that means cow. In this condition, the meaning of the old items was retained. In the inconsistent condition, the old items consisted of pictorial images of butterflies during the preview. When the new items, Japanese kanji characters meaning $d o g$, appeared at blank locations, the old items simultaneously turned into the Japanese kanji character for cow. In these conditions, the participants searched for a tilted kanji character meaning $\operatorname{dog}$ and indicated the tilt. If the advantage offered by the consistency in meaning observed in Experiment 1A was limited to cases in which the items before and after the change differed slightly, search efficiency across the three conditions would be comparable. That is, no visual marking would be obtained in the present circumstance, because the items before and after shared no figural or conceptual similarities; the relationship between the two instances would be arbitrary. In contrast, if the visual system could exploit semantic consistency for visual marking, search efficiency in the consistent condition would be higher than that in the other two conditions.

\section{Method}

A new group of 21 undergraduate students (18 males, 3 females; mean age, 21.8 years) from the AIST participant pool participated for pay. None had participated in the previous experiments. All reported normal or corrected-to-normal visual acuity and normal color vision.

The stimuli, apparatus, and procedure were the same as those in Experiment 1A, with the following exceptions. The stimuli consisted of grayscale pictures of cows and butterflies (all subtending $1.14^{\circ}$ in height and $1.8^{\circ}$ in width) taken from the colored and shaded images set of "Snodgrass and Vanderwart-like" objects (Rossion \& Pourtois, 2004) and gray Japanese kanji characters representing cow and $d o g$, each subtending $0.9^{\circ}$ in height and $1.8^{\circ}$ in width. The target was a tilted $\left(15^{\circ}\right.$ or $345^{\circ}$ from the vertical) kanji character for $d o g$, whereas the distractors were upright kanji characters for $\operatorname{dog}$ and tilted kanji characters for cow. The preview items were upright pictures of cows and butterflies. These items were presented at randomly selected locations on an invisible $5 \times 5$ matrix subtending $8.05^{\circ}$ in height and $11.5^{\circ}$ in width (see Figure 5). In the consistent condition, the old items (pictures of cows) were presented for $1,000 \mathrm{msec}$, and then each was replaced with a kanji character for cow. In the inconsistent condition, the old items (pictures of butterflies) were replaced by kanji characters for cow. Upon these changes, new items (kanji characters for $d o g$ ) appeared at blank locations. In the control condition, characters for cow and $d o g$ appeared simultaneously. On these trials, the participants searched for a tilted kanji character meaning $d o g$ and indicated the tilt by pressing the " $z$ " (for a tilt to the left) or the " $m$ " (for a tilt to the right) key.

\section{Results}

Figure 6 shows the mean reaction times. Table 1 shows the mean error rates, and Table 2 shows the associated search slopes and intercepts. An ANOVA on the reaction time data, with meaning (consistent, inconsistent, and control) and set size (4 and 16) as within-subjects factors, identified significant main effects of meaning $[F(2,40)=$ $12.34, p<.01]$ and set size $[F(1,20)=247.78, p<.01]$. The interaction between these factors was also significant $[F(2,40)=8.26, p<.01]$. A similar analysis on the error rates indicated that the main effect of set size was significant $[F(1,20)=9.57, p<.01]$. No other main effects or interactions were found. To compare the three search types more closely, we conducted three separate two-way within-subjects ANOVAs contrasting the preview conditions with each other and with the control condition.

Consistent versus control. The main effects of meaning and set size were significant $[F(1,20)=15.54, p<$ .01 , and $F(1,20)=243.66, p<.01$, respectively]. The interaction between these factors was also significant $[F(1,20)=12.79, p<.01]$. 


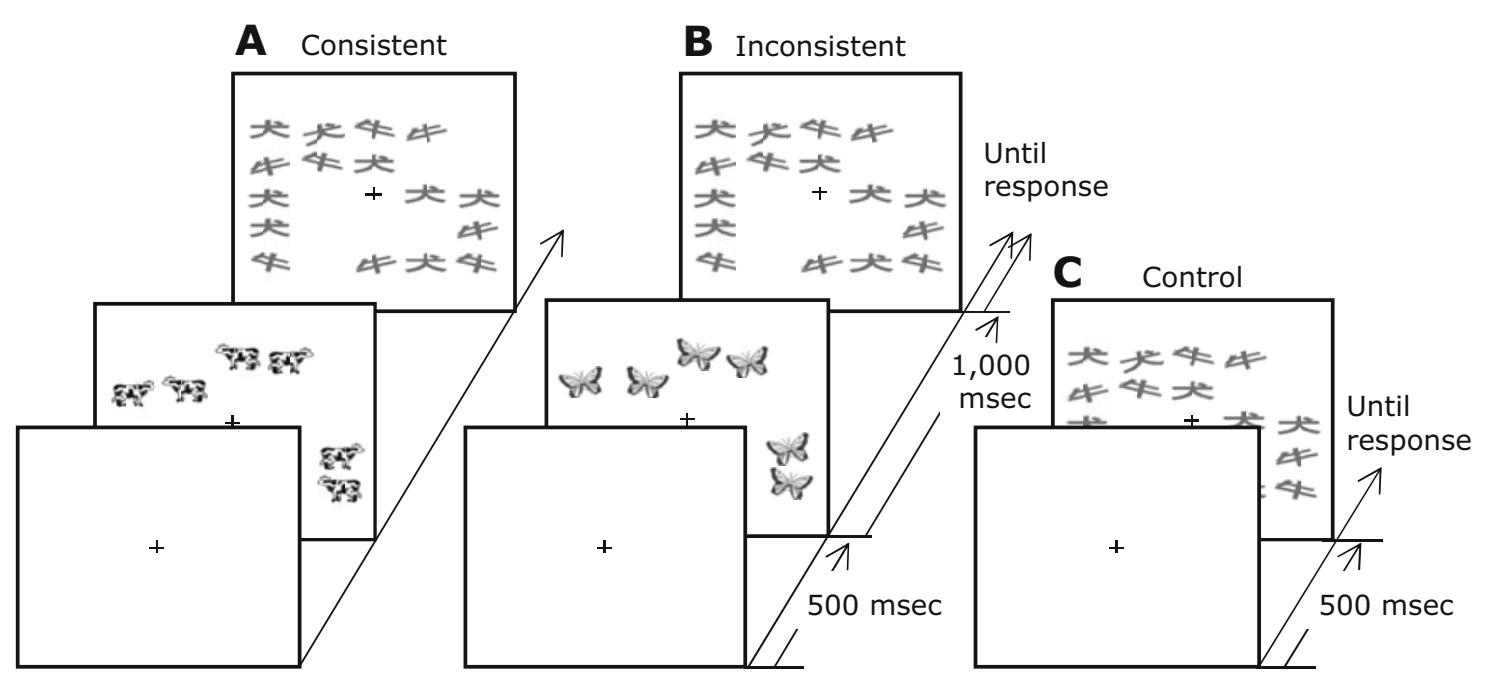

Figure 5. Schematic diagrams of stimulus sequences in Experiment 2A. (A) The consistent condition. The old items (pictures of cows) were replaced with Japanese kanji characters for cow, and new items (kanji characters that mean dog) appeared. (B) The inconsistent condition. The old items (pictures of butterflies) were replaced with Japanese kanji characters for cow, and new items (kanji characters for $\mathrm{dog}$ ) appeared. (C) The control condition. All items (kanji characters for cow and $\mathrm{dog}$ ) appeared simultaneously.

Inconsistent versus control. The main effect of set size was significant $[F(1,20)=273.67, p<.01]$. The main effect of meaning and the interaction between these factors were not significant $[F(1,20)=0.46, p=.51$, and $F(1,20)=0.34, p=.57$, respectively].

Consistent versus inconsistent. The main effects of meaning and set size were significant $[F(1,20)=19.29$, $p<.01$, and $F(1,20)=178.85, p<.01$, respectively]. The interaction between these factors was also significant $[F(1,20)=9.94, p<.01]$.

\section{Discussion}

The slope in the inconsistent condition was comparable to that in the control condition, indicating that visual marking was destroyed by the graphical and semantic changes introduced in the present experiment. More important, we replicated the advantage of consistency in meaning across the old and new items: The search slope in the consistent condition was shallower than those in the inconsistent and control conditions. These results indicate that visual marking was partially preserved when the old items changed graphically during the search if the semantic identity was consistent. This finding is consistent with the notion that visual marking is not eliminated by graphical change as long as the meaning is maintained, and it precludes the hypothesis that the advantage deriving from meaning consistency is limited to trivial figural changes. These results suggest that the memory template that underlies visual marking retains the semantic identities of old items.

The present results differed from those of Experiments $1 \mathrm{~A}$ and $1 \mathrm{~B}$, in which the slope in the inconsistent condition was shallower than that in the control condition. One possible explanation for this difference between experiments is that the graphical identities of old items in the inconsistent condition were maintained in Experiments 1A and $1 \mathrm{~B}$ because the stimulus changes used in those experiments were smaller, relative to the change used in Experiment 2A. Thus, trivial differences in the memory representation in the template, if any, may have been neglected or altered to bridge the states before and after the changes in Experiments 1A and 1B. However, given the absence of

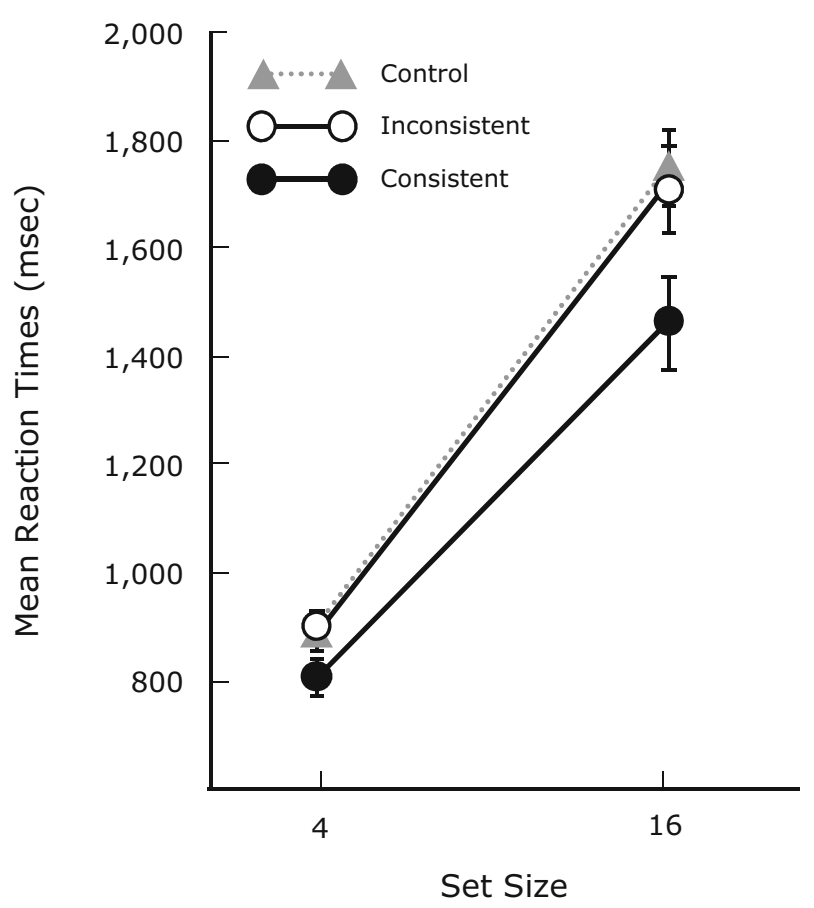

Figure 6. Mean reaction times for visual search as a function of set size in the consistent, inconsistent, and control conditions in Experiment 2A. 
Table 2

Search Function Statistics in Experiments 1-4

\begin{tabular}{clcc}
\hline \multirow{2}{*}{ Experiment } & & \multicolumn{2}{c}{ Set Size } \\
\cline { 3 - 4 } 1A & Condition & Slope (msec/Item) & Intercept (msec) \\
\cline { 3 - 4 } 1B & Consistent & 28.0 & 560.8 \\
& Inconsistent & 35.7 & 534.7 \\
& Control & 47.4 & 530.8 \\
& Consistent & 28.4 & 651.0 \\
& Inconsistent & 35.5 & 603.0 \\
& Control & 51.6 & 581.1 \\
& Consistent & 54.1 & 596.6 \\
& Inconsistent & 68.3 & 619.4 \\
2B & Control & 70.6 & 622.5 \\
& Consistent & 66.6 & 565.0 \\
& Inconsistent & 61.5 & 600.1 \\
3 & Control & 67.4 & 607.3 \\
& Consistent & 57.8 & 644.0 \\
& Inconsistent & 69.9 & 685.6 \\
& No change & 34.5 & 634.4 \\
\multirow{2}{*}{4} & Control & 68.3 & 713.0 \\
& Consistent & 55.4 & 765.5 \\
& Inconsistent & 70.6 & 697.3 \\
& Control & 66.7 & 689.1 \\
\hline
\end{tabular}

any effect of visual marking in the inconsistent condition in the present experiment, it is assumed that sufficiently large changes, such as those from the picture image of a butterfly to the kanji character for cow, can signal resetting the memory template during search.

One remaining issue to be discussed is inconsistency with a recent finding (Watson et al., 2008), in which visual marking was not resistant to luminance change even though meaning was maintained when the old and new items shared the same color. Specifically, Watson et al. (2008) used a set of capital letters (Experiment 3 ) or rotated $T_{\mathrm{s}}$ (Experiment 4) as search items. Critically, their old and new items did not differ semantically; thus, meaning-based grouping and segmentation were unavailable in their study. Under these circumstances, the search slope in the preview condition was identical to that in the full-baseline control condition, reflecting that fact that visual marking was destroyed by luminance change. One possible explanation for this inconsistency is that the meaning-based grouping and segmentation process that were available in the present experiments could contribute to avoiding the selection of old items over time because the old and new items differed in meaning, whereas no such grouping or segmentation was involved in Watson et al.'s (2008; Braithwaite, Humphreys, \& Hulleman, 2005) study, because the old and new items did not differ semantically. It is possible that meaningbased grouping and segmentation processes could protect the search from being disrupted by luminance change in the old items in the present experiment. As a result, visual marking would survive the luminance change when the meaning of the old item was consistent during the preview period. The present result is consistent with the idea that the inhibitory mechanism makes maximum use of available information to prioritize search among new items (Watson, Humphreys, \& Olivers, 2003). It is worth noting that an extremely strong luminance change of the old items was introduced in Watson et al.'s (2008) study, relative to that of the present study. Although such a difference in luminance may play a role, we believe that the grouping or segmentation on the basis of differences in meaning between the old and new items is a primary factor contributing to the inconsistency across the results of Watson et al. (2008) and those of the present study.

\section{EXPERIMENT 2B}

Experiment 2A replicated the effect of semantic consistency across the change. However, one could argue that this semantic effect in the preview search seems to have been an artifact of semantic priming by the old items before they changed (e.g., Meyer \& Schvaneveldt, 1971). The semantic priming hypothesis predicts that the speed of rejecting primed old distractors (i.e., the consistent condition) is facilitated, relative to that for nonprimed distractors (i.e., the inconsistent condition). To exclude the concern that some kind of priming contributes to this benefit, we conducted Experiment 2B, in which we replaced the preview display with a picture of a cow or a butterfly in the center of the display. If the benefit reflected priming, the same benefit as that in Experiment 2A would be obtained.

\section{Method}

Another sample of 22 undergraduate students (17 males, 5 females; mean age, 22.4 years) from the AIST participant pool participated for pay. The stimuli, apparatus, and procedures were the same as those in Experiment 2, with the following exceptions. In the consistent condition, a picture of a cow was presented at fixation for $1,000 \mathrm{msec}$ and then removed, at which point Japanese kanji characters for cow and $d o g$ appeared simultaneously. In the inconsistent condition, a picture of a butterfly was presented before the search. In the control condition, Japanese kanji characters for cow and dog appeared simultaneously without the preview period.

\section{Results and Discussion}

Figure 7 shows the mean reaction times. Table 1 gives the mean error rates, and Table 2 provides the associated search slopes and intercepts. The same ANOVA on the reaction time data, with meaning (consistent, inconsistent, and control) and set size (4 and 16) as within-subjects factors, indicated significant main effects of meaning $[F(2,42)=$ $3.67, p<.05]$ and of set size $[F(1,21)=309.99, p<$ .01]. However, the interaction between meaning and target locations did not reach significance $[F(2,42)=1.82, p=$ .18]. A similar analysis on the error rates revealed no main effects or interactions. The slope in the condition in which a picture preceded the search display was comparable to that in the control condition, in which no picture display appeared, regardless of whether the picture conveyed the meaning of the target (i.e., cow) or not (i.e., butterfly). This result indicates that no semantic priming occurred in the present experiment.

\section{EXPERIMENT 3}

The results thus far have revealed that visual marking is maintained when the shape of preview items changes, 


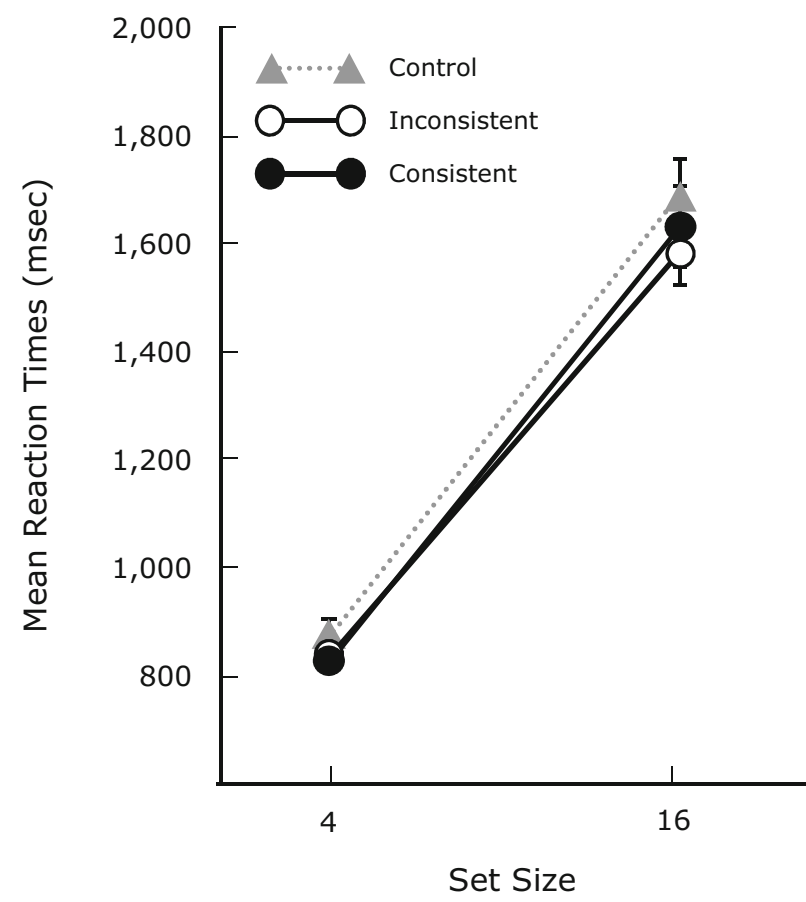

Figure 7. Mean reaction times for visual search as a function of set size in the consistent, inconsistent, and control conditions in Experiment 2B.

as long as the items are semantically consistent during the search. One remaining question is whether semantic consistency is sufficient to fully "mark" old items. Because our previous experiments did not include a baseline condition in which no change occurred in the shape and semantic domains, it was unclear whether the same degree of visual-marking effect would be obtained when mere semantic identity was maintained if shape change occurred, relative to when nothing changed. Thus, in Experiment 3, we introduced the no-change condition, in which the old items did not change when the new items appeared (akin to the standard preview or gap conditions shown by Watson \& Humphreys, 1997).

Inclusion of the no-change condition would provide a clue to understanding the mechanisms underlying visual marking. Specifically, as was noted in the introduction, we assume that visual marking is achieved by active suppression of the locations of old items via a memory template that registers graphical and semantic identities of the items. Because the participants in the present experiments could rely only on semantic identities to accomplish the current target search goal, graphical identities might not be used in the process of visual marking. From this view, consistency in semantic identities alone would be sufficient to maintain the marking of the old items in the context of the preview search. If this were the case, the slope in the consistent condition would be comparable to that in the no-change condition and shallower than those in the inconsistent and control (full-baseline) conditions. Alternatively, if semantic and graphical identities are necessary for visual marking even when the graphical identities are redundant, the slope in the consistent condition would be steeper than that in the no-change condition, although the slope in the consistent condition would remain more efficient than those in the consistent and control (full-baseline) conditions.

\section{Method}

Twenty-five undergraduate students (11 males, 14 females; mean age, 21.4 years) from the AIST participant pool participated for pay. All reported normal or corrected-to-normal visual acuity and normal color vision. None of them had participated in the previous experiments.

The stimuli, apparatus, and procedures were the same as those in Experiment 2A, except for the following changes. Each participant completed eight blocks of trials (two blocks each in the consistent, inconsistent, no-change, and control conditions). In the no-change condition, Japanese kanji characters for cow appeared in the initial display, and 1,000 msec later, the new items (kanji characters for $d o g$ ) appeared in the blank location of the initial display. In this condition, the old items did not change when the new items appeared, as in standard visual-marking studies (Watson \& Humphreys, 1997). In these conditions, the participants searched for a tilted kanji character meaning $d o g$ and indicated its tilt.

\section{Results}

Figure 8 shows the mean reaction times. Table 1 gives the mean error rates, and Table 2 presents the associated search slopes and intercepts. An ANOVA on the reaction time data, with meaning (consistent, inconsistent, no-change, and control) and set size (4 and 16) as within-subjects factors, identified significant main effects of meaning $[F(3,72)=60.47, p<.01]$ and set size $[F(1,24)=219.11$, $p<.01]$. The interaction between these factors was also significant $[F(3,72)=25.42, p<.01]$. A similar analysis

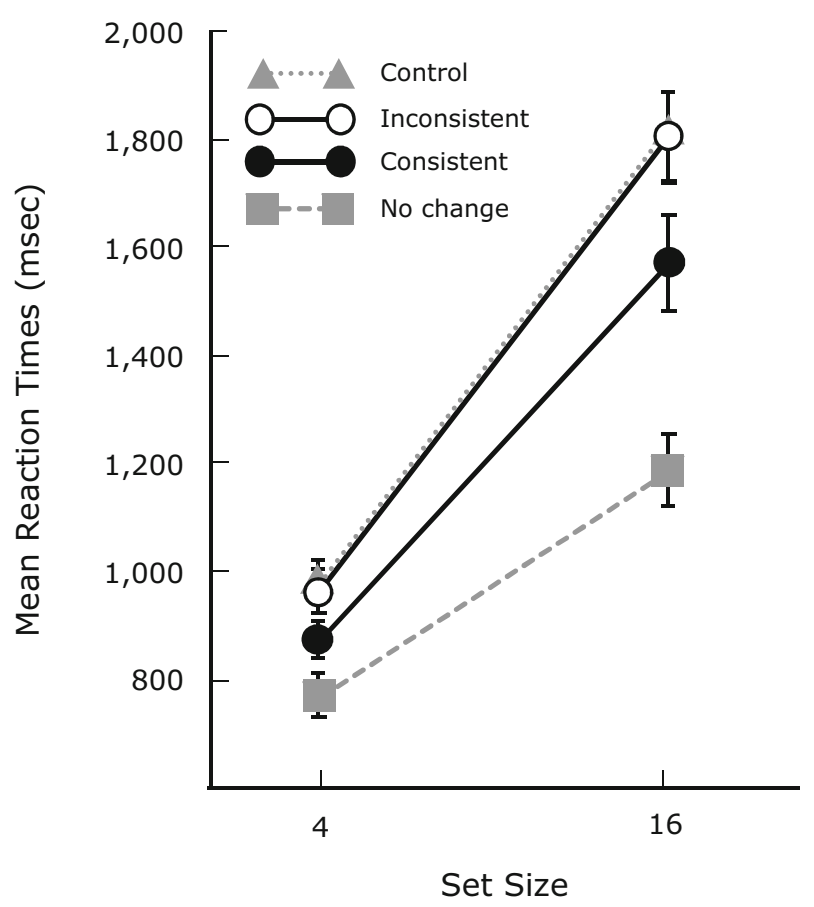

Figure 8. Mean reaction times for visual search as a function of set size in the consistent, inconsistent, no-change, and control conditions in Experiment 3. 
on the error rates indicated that the main effect of set size and the interaction between meaning and set size were significant $[F(1,24)=15.21, p<.01$, and $F(3,72)=4.09$, $p<.01$, respectively]. The main effect of meaning did not reach significance. To compare the three search types more closely, we conducted three separate two-way withinsubjects ANOVAs contrasting the preview conditions with each other and with the control condition.

Consistent versus control. The main effects of meaning and set size were significant $[F(1,24)=27.72, p<$ .01 , and $F(1,24)=194.19, p<.01$, respectively]. The interaction between these factors was also significant $[F(1,24)=5.55, p<.01]$.

Inconsistent versus control. The main effect of set size was significant $[F(1,24)=268.32, p<.01]$. The main effect of meaning and the interaction between these factors were not significant $[F(1,24)=0.11, p=.73$, and $F(1,24)=0.08, p=.78$, respectively].

Consistent versus inconsistent. The main effects of meaning and set size were significant $[F(1,24)=22.40$, $p<.01$, and $F(1,24)=162.04, p<.01$, respectively]. The interaction between these factors was also significant $[F(1,24)=8.15, p<.01]$.

Control versus no change. The main effects of meaning and set size were significant $[F(1,24)=191.01, p<$ .01 , and $F(1,24)=203.26, p<.01$, respectively]. The interaction between these factors was also significant $[F(1,24)=90.52, p<.01]$.

Consistent versus no change. The main effects of meaning and set size were significant $[F(1,24)=47.75$, $p<.01$, and $F(1,24)=129.81, p<.01$, respectively]. The interaction between these factors was also significant $[F(1,24)=22.87, p<.01]$.

Inconsistent versus no change. The main effects of meaning and set size were significant $[F(1,24)=86.70$, $p<.01$, and $F(1,24)=189.70, p<.01$, respectively]. The interaction between these factors was also significant $[F(1,24)=55.53, p<.01]$.

Errors. We also conducted three separate two-way within-subjects ANOVAs for the error rates. However, the error rate was low (4.8\% overall) and generally followed the same trends as the reaction time data.

\section{Discussion}

When the meaning was consistent, the search slope was shallower than when the meaning was inconsistent, replicating the results of Experiment 2. Furthermore, the slope in the consistent condition was steeper than that in the no-change condition. These results suggest that semantic consistency alone is insufficient to fully mark old items, although it plays a role in making search more efficient, relative to the inconsistent and control conditions. The nochange condition highlighted the joint contribution of the graphical and semantic consistencies to visual marking. In other words, when both the semantic and graphical consistencies were preserved, a full visual marking ensued (no-change condition). If graphical consistency was discontinued, the marking effect was not eliminated by the graphical change but was reduced.

\section{EXPERIMENT 4}

In Experiments 1-3, we demonstrated prioritized selection for new elements in the consistent condition, but not in the inconsistent condition. However, a concern is that the luminance changes at the old-item locations were larger in the inconsistent condition (with changes from pictures of butterflies into kanji characters for cow) than in the consistent condition (with changes from pictures of cows into kanji characters for cow). Thus, the results could be explained on the basis of this change in luminance, instead of on the basis of a difference in meaning.

To exclude this concern, we conducted Experiment 4, in which the luminance values of the pictures of a butterfly and a kanji character for cow were adjusted so that they were equal to each other. If the prioritized selection for new over old items were due to the semantic consistency of the old items, the slope in the consistent condition would be shallower than those in the inconsistent and control conditions. In contrast, if this prioritization were caused by the change in luminance, the consistent and inconsistent conditions would be comparable to each other. We also included three levels of set sizes $(4,8$, and 16) to generalize the results to other set sizes.

\section{Method}

Twenty-two undergraduate students ( 8 males, 14 females; mean age, 22.3 years) from the AIST participant pool participated for pay. All reported normal or corrected-to-normal visual acuity and normal color vision. None had participated in the previous experiments.

The stimuli, apparatus, and procedures were the same as those in Experiment 2, except for the following changes. Equiluminant pictures of a butterfly and a kanji character for cow were used (mean luminance of $0.3 \mathrm{~cd} / \mathrm{m}^{2}$, measured at the area of each picture, subtending $1.14^{\circ}$ in height and $1.8^{\circ}$ in width), and three set sizes $(4,8$, and 16$)$ were included. Thus, two independent variables were manipulated within each observation: meaning (consistent, inconsistent, and control) and set size (three levels: 4, 8, and 16). Each block consisted of a total of 60 trials ( 20 for each set size, mixed within a block). Experimental blocks were preceded by a practice block, also consisting of 60 trials.

\section{Results and Discussion}

Figure 9 shows the mean reaction times. Table 1 lists the mean error rates, and Table 2 provides the associated search slopes and intercepts. An ANOVA on the reaction time data, with meaning (consistent and inconsistent) and set size $(4,8$, and 16$)$ as within-subjects factors, identified significant main effects of meaning $[F(2,42)=3.61$, $p<.05]$ and of set size $[F(2,42)=314.47, p<.01]$. The interaction between these factors was also significant $[F(4,84)=4.34, p<.01]$. A similar analysis on the error rates revealed significant main effects of meaning $[F(2,42)=6.08, p<.01]$ and of set size $[F(2,42)=$ $5.24, p<.01]$. The interaction between these factors was not significant. To compare the three search types more closely, we conducted three separate two-way withinsubjects ANOVAs contrasting the preview conditions with each other and with the control condition. 


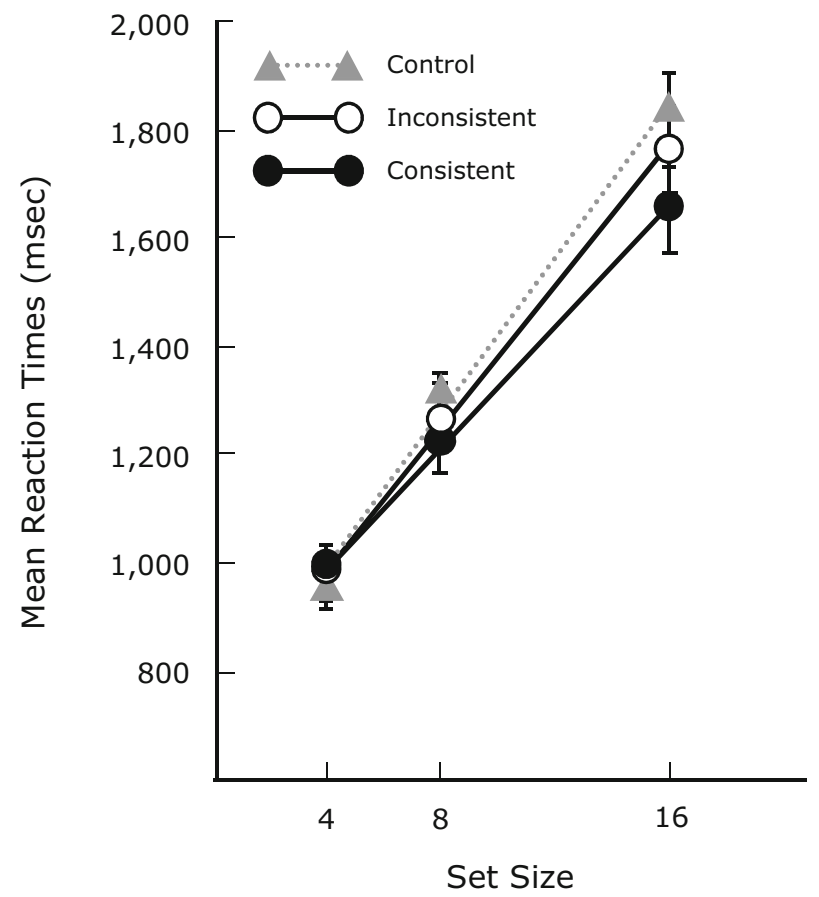

Figure 9. Mean reaction times for visual search as a function of set size in the consistent, inconsistent, and control conditions in Experiment 4.

Consistent versus control. The main effects of meaning and set size were significant $[F(1,21)=5.85, p<.05$, and $F(2,42)=300.91, p<.01$, respectively]. The interaction between these factors was also significant $[F(2,42)=$ $8.41, p<.01]$.

Inconsistent versus control. The main effect of set size was significant $[F(2,42)=287.16, p<.01]$. The main effect of meaning and the interaction between these factors were not significant $[F(1,21)=1.74, p=.20$, and $F(2,42)=0.61, p=.55$, respectively].

Consistent versus inconsistent. The main effect of meaning did not reach significance $[F(1,21)=2.33, p=$ .14]. The main effect of set size was significant $[F(2,42)=$ $216.73, p<.01]$. The interaction between these factors was also significant $[F(2,42)=5.63, p<.01]$.

The pattern of results is consistent with those from Experiments $2 \mathrm{~A}-3$, suggesting that visual marking is obtained through semantic consistency of the old items and excluding the alternative hypothesis of luminance differences.

\section{GENERAL DISCUSSION}

The present study examined the contents of the memory template that are responsible for visual marking by asking whether visual marking is preserved when graphical change occurs but semantic identity is maintained. Experiments 1 and $2 \mathrm{~A}$ revealed that this was the case. The search functions remained relatively efficient across graphical changes in old items as long as their meaning was maintained. Experiments 2B and 4 excluded the possibilities that the results of the first two experiments were due to either semantic priming or changes in luminance. These results suggest that the memory template responsible for visual marking represents semantic identity. Consistent semantic identity supports maintenance of visual marking in such memory representation during the preview period, even when graphical identity is not preserved.

In Experiment 3, we tested whether semantic consistency alone was sufficient for the prioritization of new items over old items. The result replicated the preservation of visual marking across graphical change, but visual marking was reduced by change in shape. This result suggests that semantic consistency alone was insufficient to maintain visual marking, and the memory template included the graphical and semantic representations of old items.

The present study extends the idea that the contents of the memory template reflect current visual input and that the template is updated whenever any changes occur in that input (Watson \& Humphreys, 1997). Previous studies have emphasized the importance of consistency in graphical identities for visual marking to be maintained. For example, the visual-marking effect persisted when the color or luminance of old items was changed, but the effect disappeared when the shape of the old items changed (Watson \& Humphreys, 2002). This finding suggests that visual change in the graphical identities of old items signals the memory template to reset. If a signal is elicited by a graphical change in the visual input, the signal induces discontinuation of the memory template. However, previous studies confounded changes in graphical identity with changes in semantic identity, because the two identities covaried in the stimuli that were used. The present study dissolved this confounding by introducing graphical changes without semantic changes and revealed that the template is discontinued unless the semantic identity is maintained. The present results do not necessarily mean that any graphical change induces discontinuation of the memory template. When the luminance or motion changes accompanying the graphical change were subtle (e.g., from the letter $f$ to $T$, as in Experiment 1A) and when the graphical change was irrelevant to the target's shape (see the Discussion section in Experiment 1B), the visual marking was not abolished, suggesting that the memory template is not destroyed by such a minor or irrelevant change. The present study suggests that the memory template is destroyed when a substantial change occurs in the graphical identity and in the semantic identity.

As was noted previously, it has been suggested that the memory template underlying visual marking is modified by bottom-up activities (e.g., transient signals due to stimulus onset or change) at the location of old items. The present study suggests that such bottom-up signals can be disabled by top-down control. To be specific, we assume that the visual system monitors semantic consistencies in the contents of the memory template. As long as semantic consistency is maintained, the system protects the template in a top-down manner from bottom-up signals that would otherwise trigger destruction of the memory template and reprioritize the locations where changes oc- 
curred. If the semantic identities in the memory template have been changed, the system accepts the bottom-up signal to destroy the template and selects the locations where changes occurred.

The present results cannot be explained by the proposal that visual marking is primarily due to attentional capture by newly appearing items (Donk \& Theeuwes, 2001) or to the temporal grouping of commonly appearing items (Jiang et al., 2002b), for the following reason. In the present study, transient change occurred in the old items when new items appeared. Therefore, the prioritization of new over old items should not have occurred in the present circumstance, since both stimulus onset and stimulus offset occurred for the old items (Donk \& Theeuwes, 2001; Pratt et al., 2007) when the new items appeared (Jiang et al., 2002b).

In conclusion, the present study reveals that visual marking is not destroyed by a subtle or irrelevant graphical change. Moreover, visual marking survives graphical changes in old items as long as their semantic identity is maintained. The present results suggest that the memory template contributing to visual marking represents the semantic identity of old items. We propose that bottomup signals that are elicited by graphical change and that lead to destruction of the memory template can be canceled by top-down processes that monitor consistency in the contents of the memory template and permit memory trace destruction only when semantic consistency is breached.

\section{AUTHOR NOTE}

Correspondence concerning this article should be addressed to T. Osugi, Department of Psychology, Chukyo University, 101-2 Yagotohonmachi, Nagoya, 466-8666, Japan (e-mail: mtaka-oosugi@aist .go.jp).

\section{REFERENCES}

Agter, F., \& Donk, M. (2005). Prioritized selection in visual search through onset capture and color inhibition: Evidence from a probe-dot detection task. Journal of Experimental Psychology: Human Perception \& Performance, 31, 722-730.

Brainard, D. H. (1997). The Psychophysics Toolbox. Spatial Vision, 10, 433-436.

Braithwaite, J. J., \& Humphreys, G. W. (2003). Inhibition and anticipation in visual search: Evidence from effects of color foreknowledge on preview search. Perception \& Psychophysics, 65, 213-237.

Braithwaite, J. J., Humphreys, G. W., \& Hulleman, J. (2005). Colorbased grouping and inhibition in visual search: Evidence from a probe detection analysis of preview search. Perception \& Psychophysics, 67, 81-101.

Donk, M. (2005). Prioritizing selection of new elements: On the time course of the preview effect. Visual Cognition, 12, 1373-1385.

Donk, M., Agter, F., \& Pratt, J. (2009). Effects of luminance change in preview search: Offsets and onsets can be concurrently prioritized but not in isolation. Acta Psychologica, 130, 260-267.

Donk, M., \& Theeuwes, J. (2001). Visual marking beside the mark: Prioritizing selection by abrupt onsets. Perception \& Psychophysics, 63, 891-900.

Donk, M., \& Theeuwes, J. (2003). Prioritizing selection of new elements: Bottom-up versus top-down control. Perception \& Psychophysics, 65, 1231-1242.

Donk, M., \& Verburg, R. C. (2004). Prioritizing new elements with a brief preview period: Evidence against visual marking. Psychonomic Bulletin \& Review, 11, 282-288.
Egeth, H. E., Virzi, R. A., \& Garbart, H. (1984). Searching for conjunctively defined targets. Journal of Experimental Psychology: Human Perception \& Performance, 10, 32-39.

JiAng, Y., Chun, M. M., \& Marks, L. E. (2002a). Visual marking: Dissociating effects of new and old set size. Journal of Experimental Psychology: Leaning, Memory, \& Cognition, 28, 293-302.

JiAng, Y., Chun, M. M., \& Marks, L. E. (2002b). Visual marking: Selective attention to asynchronous temporal groups. Journal of Experimental Psychology: Human Perception \& Performance, 28, 717-730.

Kaptein, N. A., Theeuwes, J., \& van der Heijden, A. H. C. (1995). Search for a conjunctively defined target can be selectively limited to a color-defined subset of elements. Journal of Experimental Psychology: Human Perception \& Performance, 21, 1053-1069.

Kunar, M. A., Humphreys, G. W., \& Smith, K. J. (2003a). History matters: The preview benefit in search is not onset capture. Psychological Science, 14, 181-185.

Kunar, M. A., Humphreys, G. W., \& Smith, K. J. (2003b). Visual change with moving displays: More evidence for color feature map inhibition during preview search. Journal of Experimental Psychology: Human Perception \& Performance, 29, 779-792.

Kunar, M. A., Humphreys, G. W., Smith, K. J., \& Watson, D. G. (2003). When a reappearance is old news: Visual marking survives occlusion. Journal of Experimental Psychology: Human Perception \& Performance, 29, 185-198.

Meyer, D. E., \& Schvaneveldt, R. W. (1971). Facilitation in recognizing pairs of words: Evidence of a dependence between retrieval operations. Journal of Experimental Psychology, 90, 227-234.

Olivers, C. N. L., \& Humphreys, G. W. (2002). When visual marking meets the attentional blink: More evidence for top-down, limitedcapacity inhibition. Journal of Experimental Psychology: Human Perception \& Performance, 28, 22-42.

Osugi, T., Kumada, T., \& Kawahara, J. (2009). The spatial distribution of inhibition in preview search. Vision Research, 49, 851-861.

Pelli, D. G. (1997). The VideoToolbox software for visual psychophysics: Transforming numbers into movies. Spatial Vision, 10, 437-442.

Posner, M. I. (1980). Orienting of attention. Quarterly Journal of Experimental Psychology, 32, 3-25.

Pratt, J., Theeuwes, J., \& Donk, W. (2007). Offsets and prioritizing the selection of new elements in search displays: More evidence for attentional capture in the preview effect. Visual Cognition, 15, 133-148.

Rossion, B., \& Pourtois, G. (2004). Revisiting Snodgrass and Vanderwart's object pictorial set: The role of surface detail in basic-level object recognition. Perception, 33, 217-236.

Sobel, K. V., \& CAVE, K. R. (2002). The roles of salience and strategy in conjunction search. Journal of Experimental Psychology: Human Perception \& Performance, 28, 1055-1070.

Theeuwes, J. (1992). Perceptual selectivity for color and form. Perception \& Psychophysics, 51, 599-606.

TheEuwes, J. (2004). Top-down search strategies cannot override attentional capture. Psychonomic Bulletin \& Review, 11, 65-70.

Watson, D. G., Braithwaite, J. J., \& Humphreys, G. W. (2008). Resisting change: The influence of luminance changes on visual marking and the preview benefit. Perception \& Psychophysics, 70, 1526-1539.

Watson, D. G., \& Humphreys, G. W. (1997). Visual marking: Prioritizing selection for new objects by top-down attentional inhibition of old objects. Psychological Review, 104, 90-122.

Watson, D. G., \& Humphreys, G. W. (2000). Visual marking: Evidence for inhibition using a probe-dot detection paradigm. Perception \& Psychophysics, 62, 471-481.

Watson, D. G., \& Humphreys, G. W. (2002). Visual marking and visual change. Journal of Experimental Psychology: Human Perception \& Performance, 28, 379-395.

Watson, D. G., \& Humphreys, G. W. (2005). Visual marking: The effects of irrelevant changes on preview search. Perception \& Psychophysics, 67, 418-434.

Watson, D. G., Humphreys, G. W., \& Olivers, C. N. L. (2003). Visual marking: Using time in visual selection. Trends in Cognitive Sciences, 7, 180-186.

Watson, D. G., \& InGLIS, M. (2007). Eye movements and time-based selection: Where do the eyes go in preview search? Psychonomic Bulletin \& Review, 14, 852-857. 
WolfE, J. M. (1994). Guided Search 2.0: A revised model of visual search. Psychonomic Bulletin \& Review, 1, 202-238.

Wolfe, J. M., Butcher, S. J., Lee, C., \& Hyle, M. (2003). Changing your mind: On the contributions of top-down and bottom-up guidance in visual search for feature singletons. Journal of Experimental Psychology: Human Perception \& Performance, 29, 483-502.

YAnTIS, S., \& Jones, E. (1991). Mechanisms of attentional selection: Temporally modulated priority tags. Perception \& Psychophysics, 50, 166-178.

Yantis, S., \& Jonides, J. (1984). Abrupt visual onsets and selective attention: Evidence from visual search. Journal of Experimental Psychology: Human Perception \& Performance, 10, 601-621.
Yantis, S., \& Jonides, J. (1990). Abrupt visual onsets and selective attention: Voluntary versus automatic allocation. Journal of Experimental Psychology: Human Perception \& Performance, 16, 121-134.

\section{NOTE}

1. As used here, graphical refers to the various physical features that contain the luminance, color, shape, and so on.

(Manuscript received May 25, 2009;

revision accepted for publication June 8, 2010.) 